\section{FRI0280 BAFF AND APRIL GENE EXPRESSION IN PATIENTS WITH ANCA ASSOCIATED VASCULITIS}

Saket Jha ${ }^{1}$, Jagdeep Singh ${ }^{2}$, Ranjana Minz ${ }^{2}$, Aadhar Dhooria', Gsrsnk Naidu', Rajiv Kumar ${ }^{1}$, Manish Rathi ${ }^{3}$, Sanjay Jain ${ }^{1}$, Shashi Anand ${ }^{2}$, Aman Sharma ${ }^{1}{ }^{1}$ Post Graduate Institute of Medical Education and Research, Chandigarh, Clinical Rheumatology and Immunology wing, Department of Internal Medicine, Chandigarh, India; ${ }^{2}$ Post Graduate Institute of Medical Education and Research, Chandigarh, Department of Immunopathology, Chandigarh, India; ${ }^{3}$ Post Graduate Institute of Medical Education and Research, Chandigarh, Department of Nephrology, Chandigarh, India

Background: Neutrophil activation is the central step in the pathogenesis of ANCA associated vasculitis (AAV). Activated neutrophils are an important source of tumor necrosis factor (TNF) ligands involved in the B cell development and survival, namely, B cell activating factor (BAFF) and a proliferating ligand (APRIL) ${ }^{1}$. They have been postulated to have a role in the pathogenesis of AAV but presently there is limited data ${ }^{2,3}$

Objectives: To study the expression of BAFF and APRIL genes in patients with AAV and healthy controls and their correlation with disease activity.

Methods: This was a prospective case-control study. Gene expression of BAFF and APRIL was studied in 20 patients of AAV (10 each with active disease and in remission) and 20 healthy age and sex matched controls. Blood samples were collected after taking due consent. Following RNA extraction (QIAamp RNA blood mini kit) and cDNA synthesis ("Thermo Scientific ${ }^{\top M}$ RevertAid ${ }^{\top M}$ First Strand" cDNA Synthesis Kit), quantitative real time polymerase chain reaction (qRT-PCR) using SYBR Green I Chemistry was carried out to study relative mRNA expression of BAFF and APRIL. $\beta$ actin (ACTB) a housekeeping gene was taken as a reference gene.

Results: Out of 20 AAV patients 16 were GPA and 4 MPA. Mean age of patients in active (8 GPA and 2 MPA) and remission (8 GPA and 2 MPA) group was $34.5 \pm 16.1$ and $39.9 \pm 16.6$ years respectively. The sex distribution in both groups was 1:1. Mean BVASv3, ESR and CRP in active AAV patients were $18.8 \pm 8.5,54 \pm 10.8 \mathrm{~mm} / \mathrm{h}$ and 41.75 (7.07- 402) $\mathrm{mg} / \mathrm{l}$ respectively. BAFF gene expression was significantly higher in both active AAV group and remission AAV group compared to controls $(p<0.01$, Figure 1$)$. The BAFF expression was significantly higher in AAV patients in remission compared to active AAV patients $(p=0.008)$. In contrast, the APRIL expression did not differ between AAV patients and controls $(P=0.829)$ or between active and inactive AAV patients $(p=0.166)$. There was no significant correlation of both BAFF and APRIL expression with disease activity markers (ESR, CRP, platelets and BVASv3).

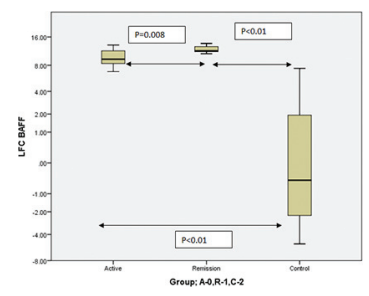

Figure 1. Box and whisker plot of BAFF expression in active AAV patients, AAV in remission and controls

Fig 1- $\mathrm{X}$ axis shows the patients groups $\mathrm{A}-0$ (active), $\mathrm{R}-1$ (remission), C-2(control); $Y$ axis shows the log fold change (LFC) of BAFF expression

Conclusion: BAFF gene is significantly expressed in patients with ANCA associated vasculitis. Among AAV patients there is significantly higher expression of BAFF in patients with inactive disease than active disease. There is no significant APRIL gene expression in patients with AAV.

\section{REFERENCES:}

[1] Mackay F, Schneider P, Rennert P, Browning J. BAFF AND APRIL: a tutorial on B cell survival. Annu Rev Immunol. 2003; 21:231-64.

[2] Krumbholz M, Specks U, Wick M, Kalled SL, Jenne D, Meinl E. BAFF is elevated in serum of patients with Wegener's granulomatosis. J Autoimmun. 2005; 25:298-302.

[3] Schneeweis C, Rafalowicz M, Feist E, Buttgereit F, Rudolph PE, Burmester GR, et al. Increased levels of BLyS and SVCAM-1 in anti-neutrophil cytoplasmatic antibody (ANCA)-associated vasculitides (AAV) Clin Exp Rheumatol. 2010; 28:62-6.

Disclosure of Interests: None declared

DOI: 10.1136/annrheumdis-2019-eular.2217

\section{FRI0281 \\ MACRO AND MICRO-VASCULAR AFFECTION IN CORRELATION TO HLA-B51 IN EGYPTIAN PATIENTSWITH BEHÇET'S DISEASE}

Manal Elmeniawy ${ }^{1}$, Mary Fawzy ${ }^{1}$, Noha Khalii ${ }^{1}$, Mohamed Hussein ${ }^{1}$, Mervat Behiry ${ }^{1}$, Rasmia Elgohary ${ }^{1}$, Reham Abdelghany ${ }^{1}$, Samar Ali ${ }^{1}$,

Sahar Kamal ${ }^{2}$, Manal Abdelrazek ${ }^{2} .{ }^{1}$ Faculty of medicine, Cairo university, Internal Medicine, Rheumatology unit, Cairo, Egypt, ${ }^{2}$ Faculty of Medicine, Cairo university, Clinical Pathology, Cairo, Egypt

Background: Behçet's disease is a multisystem inflammatory disorder with unknown etiology.HLA-B51 antigen has been known as a genetic factor associated with $B \mathrm{D}(1)$. Vascular involvement in Behcet has been widely described to affect large \& medium vessels Vascular involvement in Behcet has been widely described to affect large \& medium vessels however, few studies demonstrated micro vascular affection through nailfold capillaroscopy examination (2).

Objectives: Objective was to highlights HLA role in different clinical presentation of $B D$, especially the vascular point of view.

Methods: 40 Egyptian Behçet's disease patients (according to the international study group of Behçet's disease, 1990), and 30 age and sex matched healthy controls where enrolled in our study. All patients were subjected to history taking and clinical examination. BDCAF, CBC, ESR, CRP, liver function and lipid profile.

Genetic typing HLA B51 by PCR, carotid intima media thickness, Capillriscopy of nail capillary fold and ankle brachial index were done to both patients and controls.

Comparison of Doppler\& capillaroscopy parameters between the studied groups

\begin{tabular}{|c|c|c|c|c|c|}
\hline & & & $\begin{array}{c}\text { Behçet's } \\
\text { group }(\mathrm{N}=50)\end{array}$ & $\begin{array}{l}\text { Control } \\
(\mathrm{N}=30)\end{array}$ & $\begin{array}{c}\mathrm{p}- \\
\text { value }\end{array}$ \\
\hline \multirow[t]{8}{*}{$\begin{array}{l}\text { Carotid } \\
\text { artery }\end{array}$} & \multirow{2}{*}{$\begin{array}{l}\text { Intima media thickness } \\
(\mathrm{mm}) \\
(\mathrm{mean} \pm \mathrm{SD})\end{array}$} & Rt & $0.09 \pm 0.12$ & $\begin{array}{c}0.07 \\
\pm 0.01\end{array}$ & $\begin{array}{c}< \\
0.001\end{array}$ \\
\hline & & Lt & $0.08 \pm 0.01$ & $\begin{array}{c}0.07 \\
\pm 0.01\end{array}$ & 0.009 \\
\hline & \multirow{2}{*}{$\begin{array}{l}\text { Peak systolic velocity } \\
(\mathrm{mm} / \mathrm{s}) \\
(\mathrm{mean} \pm \mathrm{SD})\end{array}$} & Rt & $82.49 \pm 22.82$ & $\begin{array}{c}93.46 \\
\pm 19.79\end{array}$ & 0.007 \\
\hline & & Lt & $86.24 \pm 19.65$ & $\begin{array}{c}87.85 \\
\pm 14.06\end{array}$ & 0.684 \\
\hline & \multirow[t]{2}{*}{$\begin{array}{l}\text { End diastolic volume } \\
\text { (mean } \pm S D)\end{array}$} & Rt & $27.94 \pm 10.34$ & $\begin{array}{c}37.05 \\
\pm 18.18\end{array}$ & 0.002 \\
\hline & & Lt & $27.29 \pm 6.35$ & $\begin{array}{c}33.81 \\
\pm 13.48\end{array}$ & 0.021 \\
\hline & \multirow[t]{2}{*}{$\begin{array}{l}\text { Resistivity index } \\
\text { (mean } \pm \text { SD) }\end{array}$} & Rt & $0.67 \pm 0.08$ & $\begin{array}{c}0.60 \\
\pm 0.17\end{array}$ & 0.097 \\
\hline & & Lt & $0.69 \pm 0.07$ & $\begin{array}{c}0.62 \\
\pm 0.13\end{array}$ & 0.005 \\
\hline \multirow{2}{*}{\multicolumn{2}{|c|}{$\begin{array}{l}\text { Posterior tibial artery diameter }(\mathrm{mm}) \\
(\mathrm{mean} \pm \mathrm{SD})\end{array}$}} & Rt & $0.24 \pm 0.07$ & $\begin{array}{c}0.27 \\
\pm 0.08\end{array}$ & 0.079 \\
\hline & & Lt & $0.23 \pm 0.08$ & $\begin{array}{c}0.26 \\
\pm 0.08\end{array}$ & 0.083 \\
\hline \multirow{2}{*}{\multicolumn{2}{|c|}{$\begin{array}{l}\text { Anterior tibial artery diameter }(\mathrm{mm}) \\
(\text { mean } \pm \text { SD) }\end{array}$}} & Rt & $0.25 \pm 0.10$ & $\begin{array}{c}0.28 \\
\pm 0.06\end{array}$ & 0.144 \\
\hline & & Lt & $0.24 \pm .0 .10$ & $\begin{array}{c}0.27 \\
\pm 0.08\end{array}$ & 0.028 \\
\hline \multirow{4}{*}{\multicolumn{2}{|c|}{ Ankle brachial index (mean $\pm S D)$}} & RT ATA & $1.20 \pm 0.25$ & $\begin{array}{c}1.21 \\
\pm 0.20\end{array}$ & 0.445 \\
\hline & & RT PTA & $1.20 \pm 0.25$ & $\begin{array}{c}1.24 \\
\pm 0.23\end{array}$ & 0.419 \\
\hline & & LT ATA & $1.18 \pm 0.22$ & $\begin{array}{c}1.19 \\
\pm 0.18\end{array}$ & 0.490 \\
\hline & & LT PTA & $1.19 \pm 0.25$ & $\begin{array}{c}1.20 \\
\pm 0.23\end{array}$ & 0.722 \\
\hline \multicolumn{2}{|c|}{$\begin{array}{l}\text { Attenuated arterial wall of tibialis } \\
\mathbf{N}(\%)\end{array}$} & & $10(20)$ & $1(2)$ & 0.004 \\
\hline \multirow[t]{11}{*}{ Capillaroscopy } & $\begin{array}{c}\text { Arterial limb (mean } \\
\pm S D \text { ) }\end{array}$ & & $10.84 \pm 3.61$ & $\begin{array}{l}12.53 \\
\pm 3.16\end{array}$ & 0.007 \\
\hline & $\begin{array}{l}\text { Posterior limb } \\
\text { (mean } \pm S D)\end{array}$ & & $16.04 \pm 6.04$ & $\begin{array}{l}17.13 \\
\pm 4.47\end{array}$ & 0.050 \\
\hline & $\begin{array}{l}\text { Capillary loop } \\
\text { (mean } \pm S D)\end{array}$ & & $19.35 \pm 6.32$ & $\begin{array}{l}22.23 \\
\pm 5.98\end{array}$ & 0.014 \\
\hline & $\begin{array}{l}\text { Capillary length } \\
(\mathrm{mm})(\mathrm{mean} \pm \mathrm{SD})\end{array}$ & & $185.79 \pm 88.60$ & $\begin{array}{l}222.17 \\
\pm 78.15\end{array}$ & 0.020 \\
\hline & $\begin{array}{l}\text { Morphology } \\
\text { N (\%) }\end{array}$ & $\begin{array}{l}\text { Abnormal } \\
\text { shape }\end{array}$ & $2(4 \%)$ & 0 & 0.044 \\
\hline & & Scanty & $3(6 \%)$ & 0 & \\
\hline & & Scarring & $3(6 \%)$ & 0 & \\
\hline & & Elongated & $5(10 \%)$ & $1(2 \%)$ & \\
\hline & & Crossed & $7(14 \%)$ & $9(18 \%)$ & \\
\hline & & Hemorrhage & $3(6 \%)$ & $6(12 \%)$ & \\
\hline & & No & $27(54 \%)$ & $34(68 \%)$ & \\
\hline
\end{tabular}

